Relations industrielles

Industrial Relations

\title{
Friedberg, Erhard, Le Pouvoir et la Règle. Dynamiques de l'action organisée
}

\section{Stéphane Le Queux}

Volume 49, numéro 2, 1994

URI : https://id.erudit.org/iderudit/050947ar

DOI : https://doi.org/10.7202/050947ar

Aller au sommaire du numéro

Éditeur(s)

Département des relations industrielles de l'Université Laval

ISSN

0034-379X (imprimé)

1703-8138 (numérique)

Découvrir la revue

Citer ce compte rendu

Le Queux, S. (1994). Compte rendu de [Friedberg, Erhard, Le Pouvoir et la Règle. Dynamiques de l'action organisée]. Relations industrielles / Industrial Relations, 49(2), 411-414. https://doi.org/10.7202/050947ar

Tous droits réservés (C) Département des relations industrielles de l'Université Laval, 1994
Ce document est protégé par la loi sur le droit d'auteur. L'utilisation des services d'Érudit (y compris la reproduction) est assujettie à sa politique d'utilisation que vous pouvez consulter en ligne.

https://apropos.erudit.org/fr/usagers/politique-dutilisation/ 
quelques années. D'ailleurs, les expériences de fausse coopération auxquelles la citation fait référence sont loin d'être aussi répandues au Québec qu'aux États-Unis et, même si on pouvait en trouver, elles ne pourraient certainement pas être utilisées pour démontrer les pratiques ayant cours actuellement dans le secteur public alors que la problématique de la coopération en est encore (au mieux) à ses premiers balbutiements.

Le dernier chapitre de cette partie concerne la gestion de la convention collective et c'est nettement le meilleur du volume. On y retrouve un contenu détaillé qui réflète bien l'expérience pratique pertinente des deux auteurs.

Le contenu de la quatrième partie, "L'avenir des relations du travail ", est excellent mais l'ordre de présentation des trois chapitres crée beaucoup de confusion. Les auteurs ont choisi de débuter avec un chapitre sur « Les nouveaux programmes de gestion des ressources humaines ", suivi d'un autre intitulé " L'adaptation aux nouvelles réalités " et, enfin, "Perspectives des relations du travail ».

En débutant cette partie par une présentation de la panoplie des programmes de gestion des ressources humaines (santé et sécurité au travail, accès à l'égalité, politiques concernant les personnes atteintes du sida, gestion de carrière, aide aux employés, gestion de la performance, participation des travailleurs, qualité de vie au travail, cercles de qualité, partenariat, communication et qualité totale), les auteurs opèrent un changement de paradigme qui prend le lecteur par surprise, d'autant plus qu'ils n'ont pas situé au début du volume la place de la GRH par rapport aux relations du travail.

Une telle omission aurait été moins lourde de conséquence pour la compréhension de la démarche des auteurs si le chapitre intitulé «Perspectives de relations du travail » avait précédé celui sur les programmes de GRH. En effet, comme ce chapitre traite de l'impact des bouleversements profonds survenus dans le contexte économique mondial sur la main-d'œuvre, l'emploi, le contexte organisationnel, la gestion et le syndicalisme, il aurait en quelque sorte servi d'entrée en matière à la description des programmes de gestion des ressources humaines qui revêtent de plus en plus d'importance au sein des entreprises.

Ce livre peut être fort utile à des personnes non familières avec le système de relations industrielles québécois et qui veulent obtenir une information générale sur ses principales composantes. Il ne peut malheureusement pas servir d'ouvrage de référence pouvant permettre à quelqu'un de se faire une opinion critique sur son fonctionnement.

Jean Borvin

Université Laval

Le Pouvoir et la Règle - Dynamiques de l'action organisée, par Erhard FRIEDBERG, Paris, Éditions du Seuil, 1993, 405 p., ISBN 2-02-019655-7.

Michel Crozier et Erhard Friedberg nous livraient en 1977, avec L'acteur et le système, une référence désormais incontournable en sociologie des organisations. Ils nous proposaient, avec l'analyse stratégique, un cadre original pour interpréter les relations sociales au travail. En étroite parenté, Le Pouvoir et la Règle pousse désormais la 
réflexion sur le "phénomène organisation », en tant que foyer de l'action collective. Ceci ne manquera pas d'éveiller l'intérêt des lecteurs déjà rôdés à l'approche stratégique. Au demeurant, l'ouvrage constitue une porte largement ouverte à tous ceux qui désirent s'y familiariser. Il s'agit ici d'éprouver l'action organisée, c'est-à-dire de considérer le jeu des acteurs dans la construction d'ordres locaux. En ces termes, action et organisation font corps. Encore et toujours pour Friedberg, l'acteur est central. Comprendre l'organisation, c'est donc dévoiler comment les acteurs gèrent leurs interdépendances stratégiques et tentent d'infléchir la structuration de leur contexte d'action. Le pouvoir et la règle ne doivent donc pas être pris concurremment mais davantage être compris dans une relation dialectique. S'attachant à l'interaction sociale comme point clé de l'analyse, l'auteur nous propose ainsi une nouvelle heuristique dont le caractère est résolument dynamique.

La structure de l'ouvrage est linéaire. Celui-ci compte quatre parties, chacune intégrant trois chapitres. Au préalable, Friedberg s'exerce à réviser la conception classique de la notion d'organisation. En rupture des approches traditionnelles qui visent à appréhender les systèmes humains dans un univers organisationnel singulier tel le taylorisme ou la bureaucratie wébérienne, il nous invite à nous affranchir de l'organisation formelle pour s'intéresser aux processus mêmes qui sont engagés dans la détermination des contextes d'action au sein desquels l'action collective s'anime. Dès lors, il ne suffit plus de penser l'organisation mais le " phénomène organisation " comme un principe général où se conjuguent action et ordre social. Retenons que Friedberg entend ainsi se détourner du clivage qui subsiste entre l'étude de l'organisation et celle de l'action collective.

Cet exercice le mène à «banaliser » l'organisation comme objet social. Les maîtres mots de la première partie sont alors relativisation et complexification. En fait, à une compréhension techniciste, instrumentale et rationnelle, il oppose une mosaïque sociale où se fondent opportunisme et contingence. La relativisation tient au caractère irréductiblement aléatoire, spécifique et innovateur de l'action, considérant aussi que l'acteur évolue selon une rationalité limitée. La complexification se justifie par la forte endogénéité des systèmes ou processus d'action organisée, où la régulation sociale, point nodal, cède une large place à l'ambiguïté.

Sans pour autant rejoindre les modèles « d'anarchie organisée » pour lesquels, en pratique, tout produit socio-organisationnel est fortuit, la réflexion soutenue est que l'organisation et ses acteurs sont loin d'être aussi déterminés et prévisibles qu'on ne le laisserait croire. L'ambition de l'auteur est-elle bien à ce titre de lever les a priori sur la notion d'organisation.

La deuxième partie est essentiellement consacrée à la nature politique des ordres locaux. La question du pouvoir y est inévitable. Friedberg le définit initialement comme "l'échange déséquilibré de possibilités d'action " (p. 113). Seulement précise-t-il que c'est d'abord la réciprocité qui prévaut, autrement dit qu'il faille reconnaître le pouvoir dans la relation d'échange qui le sous-tend, échange a fortiori négocié. En ce sens, pouvoir et coopération ne sont pas mutuellement exclusifs. Mieux encore, ils sont la conséquence naturelle l'un de l'autre. 
Autre point significatif : Friedberg replace le pouvoir dans une perspective d'action, ce qui nous renvoie aux systèmes d'action concrets où les acteurs engagent leurs ressources et exercent leurs stratégies en vue de réguler leur coopération. La médiation, attachée à la transformation ou au maintien des règles, est donc soumise à une régulation mixte. Le pouvoir transparaît ainsi dans sa dimension collusoire.

Une telle visée conduit l'auteur à considérer la question de la formalisation dans la structuration sociale des champs d'action, puis le rapport entre ordre local et régulation globale. La première réfère principalement aux tensions existantes entre les règles du jeu et les jeux des acteurs dans la définition ou refonte de ces dernières. L'examen du second illustre avec force le leitmotiv de l'ouvrage. En effet, puisque l'organisation est révélée comme un construit politique, l'hypothèse retenue est celle d'une contingence radicale des systèmes d'action. Certes, il y a interférence entre les phénomènes d'ordres locaux et les systèmes de régulation globale, sauf que l'approche organisationnelle conserverait pour priorité leur traduction locale.

La troisième et la dernière parties traitent de l'approche organisationnelle, successivement comme principe heuristique et outil de recherche. Autre qu'une théorisation substantive de l'organisation, pour laquelle d'ailleurs il demeure largement critique, Friedberg soumet une démarche qui se veut davantage interprétative, ouverte à une compréhension dynamique de l'action organisée.

Relativement au raisonnement sur l'organisation, plusieurs mises en garde ressortent. D'abord, il est question de l'usage de la notion d'acteurs. Friedberg observe qu'il ne s'agit pas là d'une notion floue, mais bien de réalités empiriques, d'individus. De surcroît, il rappelle qu'il est indispensable de reconnaître qu'à différents degrés, nous faisons face à des acteurs intéressés. C'est ainsi qu'il introduit le concept « d'utilitarisme ". Ce dernier a surtout une valeur méthodologique, et ce, pour répondre à une double exigence : celle de contextualiser de façon systématique l'acteur et son action dans un système concret de relations, et celle d'en relever les enjeux.

Le pouvoir trouve ici une place éminemment centrale. Cependant, Friedberg nous prévient de toute interprétation machiavéliste de l'organisation en ne traduisant pas le pouvoir comme la motivation première des acteurs mais plutôt en termes de capacités d'action. De telles positions ont de fortes implications pour la construction théorique et la recherche. En effet, selon Friedberg, la pertinence de la théorie viendrait de sa capacité à se renvoyer sans cesse au terrain. En pratique, nous entrons là dans le domaine de la recherche-action, ce qui réclame fondamentalement une posture d'induction. Clairement, l'attrait de la démarche est son pragmatisme.

La production de la connaissance sur l'organisation se ferait donc à la croisée de l'intervention du chercheur et de la compréhension même que les acteurs ont de leurs systèmes d'action. Dans cet esprit, c'est à l'appui d'une étude de cas que Friedberg exemplifie finalement quel peut être empiriquement l'apport sociologique. Il y dévoile comment les concepts à l'étude sont appropriés à l'analyse d'une réalité organisationnelle, quels rapports le sociologue peut entretenir avec son milieu, et enfin dans quelle mesure les jeux des acteurs, une fois mis en lumière, peuvent s'en trouver modifiés. On entrevoit donc une perspective de changement organisationnel, partant de l'apprentissage des acteurs, où le sociologue tient un rôle actif. Au fonds, le parti pris de l'auteur pour une " méthodologie de l'action » ne peut laisser indifférent. 
Que penser de la contribution du sociologue? Doit-il investir son terrain au point tel de promouvoir une situation nouvelle? Si une connaissance plus minutieuse du milieu passe simultanément par la révélation des jeux des acteurs, peut-il en être autrement? C'est aussi le statut théorique de la sociologie des organisations qui est questionné. Respectant l'hypothèse d'une contingence radicale des ordres locaux, devonsnous nous contenter de savoirs partiels, circonstanciels? Là réside tout le problème de la généralisation. A cet égard, Friedberg nous invite minimalement à repérer le degré de structuration des objets à l'étude, ou au possible, propose un recours à des analyses comparatives. Les lecteurs intéressés par une optique de relations industrielles pourraient soulever d'autres interrogations. Retenons en deux, liées à des thèmes centraux dans notre discipline, soit la règle et le conflit.

A vouloir rattacher au plus près le fait social de la régulation, ne risquons-nous pas paradoxalement de sous-estimer le pouvoir de la règle? Par exemple, en Amérique du Nord, l'encadrement contractuel du travail par la convention collective ne demeure$\mathrm{t}$-il pas un élément largement contraignant pour les acteurs? Vrai que la formation de cette forme de régulation (collective bargaining) rejoint historiquement une entente d'acteurs. Vrai également que la convention ne permet assurément d'apprécier quel usage en feront les acteurs. Il n'en reste pas moins que la règle détermine fortement le contexte d'action.

Friedberg lie les acteurs dans un rapport de « coopération conflictuelle ». Il s'emploie ainsi à mettre en évidence la tension relationnelle inhérente à l'exercice du pouvoir. Seulement, conflit et coopération requièrent-ils analytiquement la même attention? Même si nous admettons qu'ils forment le lot quotidien de l'activité organisationnelle, le caractère particulier de la relation d'emploi ne nous permet-il pas d'interpréter de façon plus systématique les logiques d'acteurs? Autrement dit, pouvons-nous asseoir l'analyse sur des bases matérielles (mode de production, organisation du travail, type de contrôle), sans pour autant juger a priori des formes d'accommodation issues des relations de travail? En définitive, le point sous-jacent est le lien ténu entre structure et action, le débat restant ouvert à libre appréciation.

Pour conclure, l'appel au pragmatisme et à la vigilance lancé par Friedberg risque de recueillir de nombreux suffrages. S'il nous semble qu'aujourd'hui les organisations subissent de fortes pressions normatives, il était sans doute opportun de remettre quelques pendules à l'heure. Il faut également témoigner de l'effort maintenu pour donner une nouvelle impulsion à la théorisation de l'action organisée. Cependant, si la réflexion s'inscrit en ligne directe de l'analyse stratégique, la radicalisation des propositions énoncées en 1977, soit l'extrême contingence, singularité ou autonomie confiée aux ordres locaux, nous semble discutable. Somme toute, et pour emprunter le vocabulaire de l'auteur, il s'agit d'un ouvrage « stimulant ", mais dont nous recommandons une lecture attentive.

Stéphane LE Queux 